\title{
CONHECIMENTO EM NUTRIÇÃO DE INSTRUTORES DE MUSCULAÇÃO DO MUNICÍPIO DE ARACAJU-SE
}

\author{
GR. MARCUS VINÍCIUS SANTOS DO NASCIMENTO \\ Programa de Pós-graduação em Educação Física, Centro de Ciências \\ Biológicas e da Saúde, Universidade Federal de Sergipe \\ (São Cristóvão - Sergipe - Brasil) \\ E-mail: marcusnascimentone@gmail.com
}

\section{MS. OSCAR FILIPE FALCÃO RAPOSO}

Departamento de Estatística e Ciências Atuariais, Centro de Ciências Exatas e Tecnologia, Universidade Federal de Sergipe

(São Cristóvão - Sergipe - Brasil)

E-mail: oscar.raposo@hotmail.com

\section{DR. CIRO JOSÉ BRITO}

Departamento de Educação Física e Programa de Pós-graduação em Educação Física, Centro de Ciências Biológicas e da Saúde, Universidade Federal de Sergipe (São Cristóvão - Sergipe - Brasil)

E-mail: cirojbrito@gmail.com

\author{
DRA. RAQUEL SIMÕES MENDES NETTO \\ Departamento de Nutrição e Programa de Pós-graduação em Educação \\ Física, Centro de Ciências Biológicas e da Saúde, Universidade \\ Federal de Sergipe (São Cristóvão - Sergipe - Brasil) \\ E-mail: raquelufs@gmail.com
}

\begin{abstract}
RESUMO
Este estudo teve como objetivo analisar o conhecimento em nutrição de instrutores de musculação do município de Aracaju/SE. Os participantes eram compostos por 1 I I professores e 58 estagiários e responderam um questionário com perguntas sobre alimentação, orientação nutricional e um teste sobre conhecimento em nutrição. $65 \%$ dos instrutores relataram dar orientação nutricional aos seus alunos. Os participantes responderam corretamente a 59,7\% das questões do teste de conhecimento nutricional. Os instrutores de forma geral tiveram maiores percentuais de acerto nas questões sobre Nutrição geral, do que Nutrição esportiva.
\end{abstract}

PALAVRAS-CHAVE: Conhecimento nutricional; academias de ginástica; educação fisica; nutrição. 
A preocupação com a estética corporal e, principalmente, o reconhecimento pela população da importância da atividade física para a saúde e qualidade de vida tem levado as pessoas a procurarem academias de ginástica e musculação, tornando-as um dos locais mais populares e mais procurados para se conseguir tais objetivos (ASSUMPÇÃO; DINIZ; NÚNCIO, 2007).

A nutrição adequada é de fundamental importância para que haja um melhor desempenho físico, rendimento, redução de fadiga, assim como manutenção da saúde. No entanto, devido à dificuldade que as pessoas encontram hoje em dia para se alimentar corretamente, têm crescido a comercialização e utilização de "dietas da moda" e suplementos alimentares, principalmente entre frequentadores de academias de musculação (SANTOS; SANTOS, 2002; HALLACK; FABRINI; PELUZIO, 2007).

Por estarem diretamente vinculados às academias, clubes e espaços desportivos, os profissionais e estudantes de Educação Física têm maior contato com alunos, e muitas vezes são requisitados a orientar dietas, indicar a utilização de suplementos e recursos ergogênicos, tornando-se os maiores disseminadores de informações sobre nutrição e suplementação nesses locais (PEREIRA; LAJOLO; HIRSCHBRUCH, 2003; SCOFIELD; UNRUH, 2006; HIRSCHBRUCH; FISBERG; MOCHIZUKI, 2008; MORRISON; GIZIS; SHORTER, 2004; PEREIRA; CABRAL, 2007; TORRES-MCGEHEE et al., 20 I2). No entanto, muitos não recebem a formação acadêmica necessária para isso, o que pode acabar trazendo resultados inesperados aos alunos, como por exemplo, o ganho de gordura corporal ou perda de massa magra em função de estratégias nutricionais inadequadas (TOLENTINO et al., 20 l 0).

De fato, a orientação nutricional e/ou o uso de alguns suplementos podem trazer benefícios para o atleta e até mesmo para praticantes de atividade física, entretanto, estes devem ser feitos de maneira adequada, se baseando em dados científicos e necessidades individuais, não devendo ser recomendados até que se faça a avaliação da saúde e da dieta. Nesse contexto, o nutricionista é o profissional com formação acadêmica necessária para a prescrição de tais produtos e disseminação dessas informações (HIRSCHBRUCH; FISBERG; MOCHIZUKI, 2008).

Considerando a grande quantidade de informações sobre alimentação e suplementação que são disseminadas por profissionais de educação física em academias esportivas, este trabalho teve como finalidade analisar a prática de orientação nutricional/suplementos e o conhecimento em nutrição de instrutores de musculação de academias da cidade de Aracaju/SE.

\section{CASUÍSTICA E MÉTODOS}

Foi realizado um estudo com instrutores de academias esportivas (Professor, Provisionado e Estagiário) localizadas no município de Aracaju, Sergipe. Foi definido 
como professor aquele profissional formado em educação física, bacharelado e que possuía registro no CREF (Conselho Regional de Educação Física); provisionados, os instrutores que tem experiência reconhecida antes de 1997 na área de educação física, não são bacharéis, mas receberam licença para atuar na área; e estagiário, o graduando em educação física bacharelado. Os provisionados foram incluídos no grupo de professores do estudo.

Os dados referentes ao número de academias atuantes em Aracaju que fizeram parte deste estudo foram disponibilizados pela listagem de academias registradas no Conselho Regional de Educação Física da $13^{\text {a }}$ zona em Sergipe/Bahia. De acordo com os dados referentes aos anos de 2009/20 I 0, existiam, no município de Aracaju, 7I academias de ginástica licenciadas. Destas, 12 encontravam-se na região norte, 40 na região sul, 8 na região central, 10 na região Oeste e I na região de expansão. Os critérios utilizados para a escolha das academias foram: estar registradas no CREF 13 (Conselho Regional de Educação Física da I 3 a Zona em Sergipe), oferecer atividades para ambos os gêneros, devendo possuir no mínimo a atividade de musculação.

Inicialmente, realizou-se um contato com os proprietários das academias onde foram informados sobre os propósitos e procedimentos do estudo através de uma carta de anuência, a qual autorizava a participação desta, assim como dos instrutores que desejassem ser voluntários.

Os profissionais foram abordados nas academias, de forma aleatória, em diferentes horários do dia e diferentes dias da semana, e foram informados sobre o objetivo da pesquisa, da não necessidade de identificação para responder o questionário e da garantia de anonimato. Os que desejaram participar assinaram um termo de consentimento livre e esclarecido para confirmação da participação e um breve esclarecimento do estudo.

Em seguida, foi entregue pessoalmente pelo pesquisador um questionário com perguntas sobre autoavaliação da alimentação, orientação nutricional e um teste de conhecimentos sobre nutrição.

Para selecionar, avaliar e acompanhar os sujeitos que compuseram a população de estudo, a equipe que conduziu esta pesquisa seguiu todas as normas estabelecidas na Resolução 196/1 996 sobre pesquisa envolvendo seres humanos. O projeto foi aprovado pelo Comitê de Ética e Pesquisa do Hospital Universitário/ UFS. (C.A.A.E. O 106.0. I07.000-I I ). A amostra final do estudo foi de 39 academias com 169 instrutores, sendo III professores e 58 estagiários.

\section{CÁLCULO AMOSTRAL}

Considerando que não era possível identificar previamente o número de instrutores de musculação em cada academia, utilizou-se no cálculo do tamanho 
da amostra um estudo piloto em academias do município de Aracaju, onde foi identificada a média de seis instrutores de musculação (professores/estagiários) por academia, tendo assim uma previsão total de 426 instrutores no município de Aracaju.

Considerando aqui as academias como conglomerados e baseando-se numa prevalência de 37,2\% de baixo conhecimento em nutrição (ZINN, 2004; JUZWIAK; ANCOLA-LOPEZ, 2004), e ainda assumindo um erro de dois pontos percentuais, nível de confiança de 95\% e 20\% de possíveis perdas e recusas, calculou-se necessário estudar 39 academias. Destas, foi realizada amostra estratificada proporcional por região. As academias foram divididas por sub-região através de dados da prefeitura municipal de Aracaju em zona Norte, Sul, Oeste, Central e de Expansão.

Para a seleção das academias a serem entrevistadas foi realizado sorteio aleatório, a partir de listagem numerada. No caso de recusa na participação do estudo adotou-se o critério de substituição pela academia subsequente da lista.

Para garantir a representatividade da população de instrutores de musculação no estudo, foi definida a aplicação de questionários em seis instrutores por academia (baseado no estudo piloto), sendo três professores e três estagiários. Caso a academia não apresentasse este número, buscava-se o n total de instrutores proporcionalmente (professores/estagiários).

\section{AVALIAÇÃO DOS CONHECIMENTOS SOBRE NUTRIÇÃO}

Para a avaliação dos conhecimentos sobre nutrição, foi elaborado e validado um questionário baseado no estudo de Zinn (2004), que avaliou os conhecimentos nutricionais de treinadores de rugby, e em um conduzido por Zawila, Steib e Hoogenboom (2003) para avaliar os conhecimentos sobre nutrição de corredoras colegiais. A primeira seção do questionário é formada por dados de identificação, incluindo sub-região da academia, turno de trabalho, idade, sexo, cargo na academia, escolaridade. A segunda parte do questionário compreende 13 questões envolvendo aspectos de alimentação e orientação nutricional dos participantes, perguntas sobre autoavaliação da alimentação e do conhecimento, uso de suplementos e fontes de informação sobre nutrição.

A terceira seção do questionário é o teste de conhecimentos sobre nutrição, que totaliza 33 questões, com 3 alternativas de resposta, sendo uma que está certa (valor $=1)$, outra que está errada (valor $=0$ ) e mais uma alternativa "não sei" (valor =0). A primeira seção do teste foi formada por I I questões relativas ao tema conhecimentos gerais de nutrição, denominada Nutrição Geral (NG), que foi dividida em duas subcategorias: Alimentos fonte, com 5 questões relativas aos alimentos que tem alto ou baixo teor de determinados nutrientes; Nutrientes: 
com seis questões relativas à função destes no corpo humano. A segunda seção do teste de conhecimentos sobre nutrição foi referente à nutrição esportiva (NE) e também foi dividida em duas subcategorias: Nutrição na atividade física, com 13 questões envolvendo a nutrição para praticantes de atividade física e atletas, e a segunda sobre Suplementação, com 9 questões sobre a utilização e função de suplementos. Inicialmente, para cada indivíduo foram definidas novas variáveis: o percentual de acertos total (pontuação geral) e o percentual de acertos em cada uma das 4 categorias: alimentos fonte, nutrientes, nutrição esportiva e suplementação.

Para análise dos conhecimentos em nutrição foram analisadas as médias de cada uma das variáveis acima referidas.

\section{VALIDAÇÃO DO QUESTIONÁRIO}

Inicialmente, para adequação da linguagem e análise dos dados, o questionário elaborado foi avaliado e aprovado por 3 pesquisadores, sendo 2 doutores na área da nutrição e com vasta experiência na área de nutrição esportiva, e o terceiro na área de ciências do esporte. Em seguida, foi conduzido um estudo piloto, onde o questionário foi testado entre 10 profissionais de educação física que não participaram do estudo.

Após esta fase, o questionário foi aplicado em 2 grupos, para determinar a validade discriminante do instrumento. $\bigcirc$ grupo I era formado por 30 estudantes do curso de exatas, enquanto o segundo, por 22 formandos e recém-formados no curso de nutrição. Foram escolhidos estes 2 grupos porque, teoricamente, os estudantes de cursos da área de exatas seriam um parâmetro de baixo conhecimento em nutrição em relação aos formandos e recém-formados em nutrição. Dessa forma, para ser considerado válido, o questionário deveria ser capaz de diferenciar os participantes em diferentes níveis de conhecimento.

Como validação discriminante, foi observada uma média de percentual de acerto significativamente maior para o grupo 2 (7 | \%), do que para o grupo I (4 | \%). A replicabilidade foi aferida em 15 formandos ou recém-formados em nutrição, que responderam o questionário em um intervalo de até I mês. A correlação entre as respostas das 2 aplicações foi significante e moderadamente alta $(r=0,62)$.

\section{ANÁLISE ESTATIÍSTICA}

Para análise estatística foi utilizado o software SPSS, versão 19 (SPSS Inc., Chicago, IL). Em relação ao questionário utilizado, a verificação da consistência interna, ou seja, o grau pelo qual as questões de cada subcategoria são coesivas e homogêneas em conteúdo, foi obtido por intermédio do coeficiente de fidedignidade de Alfa Cronbach ( $\alpha$ ). Conforme Streiner (2003), este coeficiente está sendo 
amplamente utilizado para atestar a confiabilidade das respostas obtidas em análises realizadas através de questionários. Sua variabilidade está compreendida entre 0,00 (ausência de confiabilidade) a I,00 (confiabilidade perfeita). O valor mínimo de 0,70 foi recomendado por Rowland, Arkkelin e Crisler ( $199 \mid$ ) para definir que os itens avaliam consistentemente o mesmo constructo (grupo de perguntas).

Foram utilizadas medidas de tendência central (média), variabilidade (desvio padrão) e prevalências (absoluta e relativa). Com finalidade de conferir a normalidade dos dados das variáveis contínuas foi feito o teste de Shapiro-Wilk. Devido à ausência de normalidade foi utilizado o teste não paramétrico Mann-Whitney para comparação de médias. Todos os valores de p inferiores a 5\% foram considerados estatisticamente significantes.

\section{RESULTADOS}

O valor da consistência interna das respostas do questionário foi obtido através do coeficiente de Cronbach. Os valores encontrados apresentaram fidedignidade aceitável, acima de 0,7 para professores $(0,77)$, estagiários $(0,87)$, e total $(0,83)$.

O número de participantes do estudo foi de 169 instrutores de musculação, dos quais 66\% ( $n=\mid$ II) eram professores (grupo I), enquanto 34\% $(n=58)$ eram estagiários (grupo 2). Os participantes do estudo tinham uma média e desvio padrão de $28 \pm 6,3$ anos de idade e $15 \pm 1,6$ anos de estudo, contados do ensino fundamental à pós-graduação. Foi encontrada diferença significativa $(p<0,05)$ entre as idades e a quantidade de anos de estudo dos professores e estagiários, com média de idade de $31 \pm 6$ e $24 \pm 4$ anos, e de $15 \pm 1,7$ e $14 \pm 0,8$ anos de estudo, respectivamente.

A maioria dos instrutores (74\%) relatou que considera a sua alimentação boa, mas pode melhorar, 15\% relataram considerar sua alimentação muito boa ou ótima. Com relação aos instrutores que relataram ter uma alimentação ruim ( 1 1\%), a maior parte destes referiu como principal motivo a falta de tempo (72\%).

Analisando o uso de suplementos, aproximadamente metade da população $(50,3 \%)$ relatou fazer o uso destes, considerando os últimos 12 meses. Com relação à autoavaliação do conhecimento sobre nutrição, a maioria classificou seu conhecimento em nutrição em mediano/ruim (51,5\%).

A maior fonte de busca de informações sobre nutrição, relatada pelos participantes, foram palestras/seminários/cursos (72,7\%), seguida de internet (65\%) e revistas $(41,4 \%)$.

$\mathrm{Na}$ Tabela I estão descritos os dados referentes à orientação nutricional concedida. A maior parte dos instrutores relatou dar orientação nutricional aos seus alunos, sendo os principais aspectos inclusos a ingestão de alimentos antes do 
treino, seguida da ingestão de alimentos após o treino e ingestão de líquidos. Dos participantes que referiram não fornecer orientação nutricional aos seus alunos, o principal motivo relatado foi o fato de não ser função do profissional, seguido de não tenho confiança no meu nível de conhecimento nutricional.

Tabela I. Aspectos relacionados à orientação nutricional dos instrutores de musculação do município de Aracaju, Sergipe, 201 I

\begin{tabular}{|c|c|c|}
\hline \multirow[t]{2}{*}{ Categorias } & \multicolumn{2}{|c|}{ Instrutores } \\
\hline & $\mathrm{N}$ & $\%$ \\
\hline \multicolumn{3}{|l|}{ Fornece orientação nutricional } \\
\hline $\operatorname{Sim}$ & 110 & 65 \\
\hline Não & 59 & 35 \\
\hline \multicolumn{3}{|l|}{ Por que os instrutores não fornecem orientação nutricional? } \\
\hline Falta de tempo & 4 & 6,8 \\
\hline Não tenho confiança no meu nível de conhecimento nutricional & 18 & 30,5 \\
\hline Outra pessoa dá orientação & 14 & 23,7 \\
\hline Não vejo a nutrição como um fator importante para meus alunos & I & ।,7 \\
\hline Não é minha função & 28 & 47,5 \\
\hline Outro & 2 & 3,4 \\
\hline \multicolumn{3}{|l|}{ Aspectos inclusos na orientação } \\
\hline Ingestão de líquidos & 77 & 70 \\
\hline Suplementação & 58 & 52,7 \\
\hline Ingestão de alimentos antes do treino & 96 & 87,3 \\
\hline Ganho ou perda de peso & 71 & 64,5 \\
\hline Ingestão de alimentos após o treino & 92 & 83,6 \\
\hline Tipos de nutrientes & 45 & 41,3 \\
\hline Outros & 4 & 3,7 \\
\hline
\end{tabular}

Com relação ao conhecimento em nutrição, os dados foram analisados primeiramente para identificar o tema que apresentasse o maior percentual de acerto na população, seguido da análise por tema, e subcategorias entre os grupos (professores $x$ estagiários).

O percentual de acertos dos instrutores de musculação foi maior nas questões relacionadas à nutrição geral (subcategorias alimentos fonte + nutrientes) (6I,7\%) quando comparada ao tema nutrição esportiva (subcategorias nutrição e atividade física e suplementação) $(57,7 \%)(p=0,02)$. 
Os resultados sobre o conhecimento de nutrição geral e esportiva são apresentados na Tabela 2. Os professores obtiveram nota geral e por subcategoria significativamente maiores do que os estagiários: Nota Geral $(p=0,000)$, Alimentos fonte $(p=0,000)$, Nutrientes $(p=0,0 \mid \mathrm{I})$, Nutrição e atividade física $(p=0,027)$ e Suplementação $(p=0,03 \mid)$.

Tabela 2- Médias de percentuais da pontuação total e por subcategoria dos instrutores de musculação do município de Aracaju, Sergipe, 201 l.

\begin{tabular}{|c|c|c|c|c|}
\hline Categoria & Cargo na academia & $\begin{array}{l}\text { Percentual de } \\
\text { acertos } \\
\text { Média e desvio } \\
\text { padrão }\end{array}$ & $\begin{array}{c}\text { Percentual de erros } \\
\text { Média e desvio } \\
\text { padrão }\end{array}$ & $\begin{array}{l}\text { Percentual de } \\
\text { não sei } \\
\text { Média e desvio } \\
\text { padrão }\end{array}$ \\
\hline \multirow{4}{*}{ Pontuação geral } & Professores & & & \\
\hline & & $62,5 \pm 11,4^{\mathrm{a}}$ & $26,9 \pm 20,9$ & $8,9 \pm 7,7$ \\
\hline & & $54,2 \pm 12,9^{b}$ & $29,0 \pm 21,6$ & \\
\hline & Todos & $59,7 \pm 12,5$ & $27,5 \pm 20,8$ & $\mid 1,1 \pm 8,0$ \\
\hline \multirow{3}{*}{ Alimentos Fonte } & Professores & $68,1 \pm 12,8^{a}$ & $23,9 \pm 17,4$ & $7,9 \pm 5,5$ \\
\hline & Estagiários & $55,2 \pm 15,0^{b}$ & $26,8 \pm 19,1$ & $|7| \pm 9,5$, \\
\hline & Todos & $63,7 \pm|4,9|$ & $25,1 \pm 17,4$ & $|I| \pm 6,4$, \\
\hline \multirow{3}{*}{ Nutrientes } & Professores & $62,7 \pm 21,0^{\mathrm{a}}$ & $27,7 \pm 23,1$ & $9,4 \pm 8,2$ \\
\hline & Estagiários & $53,7 \pm 20,0^{b}$ & $31,4 \pm 19,2$ & $14,7 \pm 12,5$ \\
\hline & Todos & $59,6 \pm 21,0^{2}$ & $29,0 \pm 21,6$ & $11,2 \pm 9,5$ \\
\hline \multirow{3}{*}{$\begin{array}{l}\text { Nutrição e } \\
\text { atividade física }\end{array}$} & Professores & $61,8 \pm 12,0^{\mathrm{a}}$ & $32,6 \pm 28,2$ & $5,5 \pm 7,2$ \\
\hline & Estagiários & $57,5 \pm 12,3^{b}$ & $33,0 \pm 29,7$ & $9,4 \pm 8,8$ \\
\hline & Todos & $60,3 \pm 12,3^{2}$ & $32,8 \pm 28,6$ & $6,8 \pm 7,5$ \\
\hline \multirow{3}{*}{ Suplementação } & Professores & $62,5 \pm 11,4^{\mathrm{a}}$ & $27,2 \pm 20,7$ & $17,1 \pm 10,2$ \\
\hline & Estagiários & $50,3 \pm 19,2^{b}$ & $29,3 \pm 21,1$ & $20,3 \pm 11,6$ \\
\hline & Todos & $55,0 \pm 19,8^{3}$ & $27,7 \pm 20,5$ & $17,2 \pm 10,1$ \\
\hline
\end{tabular}

a, b comparação entre professores $x$ estagiários $(p<0,05)$

1,2,3 comparação entre subcategorias, letras diferentes $(p<0,05)$ 
O questionário desenvolvido foi formado por dois temas: Nutrição Geral e Nutrição esportiva. $\bigcirc$ primeiro tema é composto pelas subcategorias Alimentos Fonte e Nutrientes, enquanto o segundo foi dividido em Nutrição e Atividade Física e Suplementação. Os resultados obtidos nas subcategorias estão descritos abaixo:

A subcategoria Alimentos fonte foi a que apresentou maior percentual de acertos. Dentre as cinco questões respondidas pelos instrutores, a que possuiu maior média de acertos estava relacionada ao conteúdo específico de gordura dos alimentos (72\%). A maior média de erros (37,74\%) foi encontrada na questão que indagava sobre o conteúdo de colesterol, enquanto que a que apresentou uma maior média de percentual de respostas "não sei", foi a número dois, a qual avaliava se os alimentos sugeridos possuíam alto ou baixo teor de proteína.

Dentro da subcategoria Nutrientes, quase todos os instrutores (92,9\%) marcaram corretamente como verdadeira a questão 10 , a qual referia que o consumo de álcool afetava a utilização e absorção dos nutrientes. O item que obteve maiores percentuais de erros como de respostas "não sei" foi o oito, o qual afirmava que no máximo I 5\% das calorias da dieta deviam ser provenientes de gordura. Neste item, mais da metade dos instrutores (54,2\%) erraram ao responder que a afirmativa era verdadeira e 26,2\% não souberam responder.

O subtema Nutrição e Atividade Física foi o que apresentou a maior quantidade de respostas incorretas. Nesta subcategoria está inclusa a questão de maior quantidade de erros do questionário de conhecimento sobre nutrição, questão 12 . Nesta, $84,6 \%$ dos instrutores erraram ao confirmar que uma pessoa fisicamente ativa e que possui uma dieta nutricional adequada pode melhorar sua performance consumindo uma maior quantidade de nutrientes. Entretanto, pertencem também a essa categoria de perguntas, as duas questões em que foram encontrados os maiores percentuais de acerto de todo o questionário de conhecimento, a 13 e a 17. $\mathrm{Na}$ questão 13, 98,8\% dos instrutores responderam que era errado pular refeições quando é preciso perder peso rapidamente, e na 17,97\% confirmou que o tipo de alimento que um atleta ingere, influencia no seu desempenho. A questão que teve maior percentual de respostas "não sei" foi a 16, a qual afirmava que algumas comidas ,por exemplo, o abacaxi, possuem grande importância em dietas para perda de peso já que poderiam ajudar na queima de gordura.

A subcategoria suplementação foi a que teve a maior quantidade de respostas "não sei". Nesta classe de perguntas, o item 25, o qual afirmava que a creatina traria mais benefícios a um atleta que queira aumentar sua força, foi o que obteve o maior número de respostas corretas, ao ser tomado por $83,4 \%$ dos instrutores como verdadeiro. A questão de número 29 foi a que teve maior percentual de erros nessa subcategoria, onde $40,8 \%$ dos instrutores afirmaram que os suplementos 
multivitamínicos deveriam ser tomados pela maioria dos atletas, mesmo consumindo uma dieta adequada e balanceada. A questão 27, a qual comentava sobre o principal mecanismo da creatina, foi a que obteve o maior percentual de respostas "não sei" (36, 1\%).

\section{DISCUSSÃO}

Dada a grande quantidade de informações sobre alimentação e nutrição disseminadas por profissionais de educação física, este estudo teve como objetivo analisar a prática de orientação nutricional/suplementos e o conhecimento em nutrição de instrutores de musculação de academias da cidade de Aracaju/SE. Resultados da pesquisa mostraram que a maior parte dos instrutores de musculação relatou fornecer orientação nutricional aos seus alunos. Além disso, apresentaram menor conhecimento em nutrição esportiva frente à nutrição geral, sendo este primeiro, o tema o qual estes profissionais emitem maior número de orientações.

A média de percentual de acertos total atingida pelos instrutores foi de 59,7\%, inferior à pontuação obtida em outros estudos, no Brasil e em diferentes países, os quais utilizaram instrumentos diferentes para avaliar o conhecimento sobre nutrição. No estudo de Rockwell, Nichols-Richardson e Thye (200I) e Marinaro (2008), onde foram avaliados os conhecimentos nutricionais de técnicos e treinadores nos Estados Unidos, o percentual de acerto foi de 67\% $(n=53)$ e 76,2\% $(n=280)$, respectivamente; semelhante ao encontrado por Torres-Mcgehee (20। 2), onde 131 técnicos e 192 preparadores físicos americanos obtiveram uma média de percentual de acerto igual a 65,9\% e 77,8\% respectivamente. No Brasil, o estudo conduzido por Perim e Zucas (200 I), que avaliaram o conhecimento nutricional de 202 professores de educação física que atuam no município de Londrina, verificou uma média de percentual de acertos de $71 \%$.

Com relação aos temas, foi encontrada diferença significativa entre a média de percentual de acerto das questões relativas à nutrição geral e nutrição esportiva $(p<0,05)$. Os instrutores de forma geral (professores e estagiários) tiveram uma média de pontuação nas questões relativas à nutrição geral significativamente maior do que à nutrição esportiva, o qual este último teve uma média de apenas metade das questões respondidas corretamente. Fato que chamou atenção, pois 65\% dos participantes forneciam orientação nutricional aos seus alunos, sendo as principais orientações referentes a aspectos relativos à nutrição no esporte, como ganho ou perda de peso, ingestão de alimentos antes e após o treino e suplementação.

Uma hipótese para explicar tal achado é que a nutrição como base sobre qual se desenvolvem todos os processos fisiológicos e patológicos, já que nenhum fenômeno orgânico normal ou anormal ocorre sem que haja um componente 
nutricional envolvido, tem uma forte ligação com outras áreas da saúde, entre elas a educação física (BOOG, 1999). Desse modo, apesar de não existirem mecanismos legais que incluam a nutrição no currículo de educação física das instituições, já que esta não é exigência para atuação profissional, a nutrição básica está presente em parte dos cursos de educação física, visto que tais conhecimentos mínimos são de fundamental importância para profissionais cujo trabalho tem relação com a promoção da saúde, prevenção de doenças ou recuperação da saúde (BOOG, 1999; JUZWIAK; ANCOLA-LOPEZ, 2004). No caso da Nutrição Esportiva, uma hipótese para explicar um menor rendimento dos instrutores em questões relativas a essa área do conhecimento, poderia ser o fato da não realização de cursos ou disciplinas na área de nutrição esportiva, apesar destes terem relatado utilizar fontes sobre informações da ciência da nutrição que oferecem maior confiabilidade, como palestras, seminários e cursos. Diferente do estudo realizado por Juzwiak e Ancona-Lopez (2004), com 55 técnicos da cidade de São Paulo, onde a principal fonte de informação sobre nutrição eram revistas não científicas, sendo estas consideradas meios inadequados de se obter tal tipo de informação (JUZWIAK; ANCONA-LOPEZ, 2004; BELTZ; DOERING, 1993).

Dessa forma, visando à melhoria das condições de saúde dos indivíduos de forma ética e segura, no ramo da nutrição compete aos profissionais de educação física apenas a disseminação de informações de cunho básico e geral sobre o tema, ato que muitas vezes é confundido com orientação nutricional, sendo este último atividade privativa do nutricionista (CFN, 2005).

A orientação nutricional ou aconselhamento dietético é mais que uma transmissão ou disseminação de informações, é uma abordagem de educação nutricional, efetuada por meio do diálogo entre o cliente portador de uma história de vida que procura ajuda para solucionar problemas de alimentação - e o nutricionista, preparado para analisar o problema alimentar no contexto biopsicossociocultural, a fim de buscar um estado de harmonia compatível com a saúde (RODRIGUES; SOARES; BOOG, 2005).

Alguns dos instrutores relataram ter conhecimento sobre tal fato, já que a maior parte daqueles que referiram não fornecer orientação nutricional a seus alunos, justificaram não ser atributo do profissional de educação física. No entanto, em um estudo conduzido por Jazayeri e Amani (2004) sobre o conhecimento nutricional, atitudes e práticas de 93 treinadores de fisiculturistas do Iran, foi verificado que todos os treinadores e $47 \%$ das treinadoras já haviam prescrito dieta aos seus atletas. Burkhart e Coad (20 I0) constataram que 62\% dos atletas adolescentes que participaram da pesquisa já haviam utilizado seu técnico como fonte de informação sobre nutrição. Além disso, Burns et al. (2004) observaram que a maioria dos 
técnicos e treinadores entrevistados acreditava que o treinador deve ser a fonte primária de informaçã̃o sobre nutrição dos atletas, e que é responsabilidade destes fornecer estas informações.

Quando os dados foram analisados por subcategoria (alimentos fonte, nutrientes, nutrição e atividade física e suplementação), foi encontrada diferença significativa entre a média de percentual de acerto encontrada em quase todas as subcategorias $(p<0,05)$. Só não foi encontrada diferença entre a média de percentual de acerto da subcategoria Nutrientes em relação à nutrição e atividade física. Dentre as subcategorias do questionário, a que teve maior quantidade de respostas "não sei" foi a suplementação, mesmo mais da metade dos instrutores tendo relatado orientar seus alunos com relação a este tema. Achados semelhantes foram encontrados em um estudo realizado por Zinn (2004) e a outro feito por Bedgood e Tuck (1983), por outro lado, técnicos e treinadores de uma universidade local tiveram 89\% de média de percentual de acerto nas questões relativas à suplementação (ROCKWELL; NICHOLS-RICHARDSON; THYE, 200I).

Em um estudo elaborado por Ropelato e Ravazzani (20 I I), sobre a percepção de acadêmicos de educação física em relação aos suplementos alimentares, 87,5\% dos entrevistados referiu ter conhecimento regular ou bom sobre a legislação pertinente, no entanto, aproximadamente metade dos alunos respondeu que o educador físico pode prescrevê-los, enquanto 4 I ,3\% relataram não conhecer os problemas do uso indiscriminado desses produtos. Isto caracteriza um quadro preocupante, visto que mostra que, além destes profissionais não receberem a formaç̧ão adequada para tal fim, muitos deles não estão cientes de como se portar diante dessas situações, onde a conduta mais adequada passa a ser o encaminhamento ao nutricionista.

Durante a análise dos dados, foi encontrada diferença significativa $(p<0,05)$ entre as médias de percentual de acerto dos professores e dos estagiários, onde os primeiros tiveram maiores valores com relação às variáveis analisadas. A média de percentual de acerto dos estagiários foi de 54\%, semelhante a um estudo realizado por Ozdogan e Ozcelik (20 I I), que avaliaram o conhecimento de 343 estudantes do departamento de educação física de três universidades na Turquia.

Também foi verificada diferença significativa $(p<0,05)$ entre a idade e anos de estudo destes dois grupos, onde os professores tinham uma maior idade e mais anos de estudo do que os estagiários. Tal achado é uma hipótese para justificar o fato de os professores terem atingido melhor média de pontuação, já que além de serem graduados e terem maior experiência, tiveram a oportunidade de participar de uma maior quantidade de palestras/seminários/cursos relacionados à nutrição, enquanto que alguns dos estagiários ainda se encontravam no início do curso de educação física. 


\section{CONSIDERAÇÕES FINAIS}

Resultados do presente estudo mostraram que a maior parte dos instrutores de musculação de academias de Aracaju (SE) relatou fornecer orientação nutricional aos seus alunos, mesmo não sendo atributo do profissional de educação física. Além disso, apresentaram menor conhecimento em nutrição esportiva do que em nutrição geral, sendo este primeiro, o tema sobre o qual estes profissionais emitem um maior número de orientações.

Nutritional Knowledge of Fitness Instructors in Aracaju-SE
ABSTRACT: The purpose of this study was to analyse the nutrition knowledge of fitness ins-
tructors in Aracaju/SE. The participants were compound of I I teachers and 58 trainees and
completed questionnaires regarding feeding, nutrition advice and nutrition knowledge. $65 \%$ of
the respondents provided nutrition advice. The participants correctly answered to $59,7 \%$ of
the nutrition knowledge questionnaire. Fitness instructors showed to have a higher percentage
of correct answers in questions related to General nutrition than in Sports nutrition..
KEYWORDS: Nutritional knowledge; Fitness Center; Physical Education; Nutrition.
Conocimiento nutricional por los instructores de
preparación física em La ciudad de Aracaju-SE
RESUMEN: El objeivo del estudio fue analizar el conocimiento de los instructores de preparación
fisica en Aracaju/SE. Los participantes eran I I I profesores y 58 pasantes, que respondieron a
preguntas acerca de la alimentación, asesoramiento nutricional y uma prueba de conocimientos
de nutrición. 65\% de los instructores dijeron que orientan sus alumnos. Los participantes
respondieron correctamente a $59,7 \%$ de las preguntas de la prueba de conocimiento de
nutrición. Los profesores, en general, tenían un mayor porcentaje de respuestas correctas a
preguntas acerca de la nutrición en general, que acerca de la nutrición deportiva.
PALABRAS CLAVE: Conocimiento nutricional; centros de acondicionamiento; educación y
entrenamiento fisico; nutrición.

\section{REFERÊNCIAS}

AMERICAN COLLEGE OF SPORTS MEDICINE, AMERICAN DIETETIC ASSOCIATION, DIETITIANS OF CANADA. Position of the American Dietetic association, Dietitians of Canada, and the American College of Sports Medicine: nutrition and athletic performance. Journal of the American Dietetic Association, Chicago, v. 109, n. 3, p. 509-527, mar. 2009.

ASSUMPÇÃO, B. V.; DINIZ, J. C.; NÚNCIO, A. A. S. O nível de conhecimento das informações sobre alimentação utilizados por indivíduos freqüentadores de academia de diferentes níveis sociais na cidade de Sete Lagoas - Minas Gerais. Revista Brasileira de Nutrição Esportiva, São Paulo, v. I, n. 5, p.01-12, set./out. 2007. 
BEDGOOD, B. L.; TUCK, M. B. Nutrition knowledge of high school athletic coaches in Texas. Journal of the American Dietetic Association, Chicago,v. 83, n. 6, p. 672-677, dec. 1983.

BELTZ, S. D.; DOERING, P. L. Efficacy of nutritional supplements used by athletes. Clinical Pharmacy, Bethesda, v. 12, n. I2, p. 900-908, dec. 1993.

BOOG, M. C. F. Dificuldades encontradas por médicos e enfermeiros na abordagem de problemas alimentares. Revista de Nutrição, Campinas, v. I 2, n. 3, p. 26 I-272, set./dez. 1999.

BURKHART, S. J.; COAD, J.; BROUGH, L. Assessment of nutrition knowledge and food skills in talented adolescent athletes. In: ANNUAL CONFERENCE OF THE NUTRITION SOCIETY OF NEW ZEALAND, 45., 2009, New Zeland. Proceedings... New Zeland, 2010.

BURNS R. D. et al. Intercollegiate student athlete use of nutritional supplements and the role of athletic trainers and dietitians in nutrition counseling. Journal of the American Dietetic Association, Chicago, v. 104, n. 2, p. 246-249, feb. 2004.

CONSELHO FEDERAL DE NUTRIÇÃO (CFN). Dispõe sobre a definição das áreas de atuação do nutricionista e suas atribuições, estabelece parâmetros numéricos de referência, por área de atuação, e dá outras providências. Resolução CFN No 380/2005. Brasília, 2005.

ERSOY, G. Egzersiz ve spor yapanlar için beslenme Ankara: Nobel Yayın Dağıtım; 2004.

HALLACK, A.; FABRINI, S.; PELUZIO, M. C. G. Avaliação do consumo de suplementos nutricionais em academias da zona sul de Belo Horizonte, MG, Brasil. Revista Brasileira de Nutrição Esportiva, São Paulo, v. I, n. 2, p. 55-60, mar./abr. 2007.

HIRSCHBRUCH, M. D.; FISBERG, M.; MOCHIZUKI, L. Consumo de suplementos por jovens frequentadores de academias de ginástica em São Paulo. Revista Brasileira de Medicina do Esporte, São Paulo,v. I4, n. 6, p. 539-546, nov./dez. 2008.

JAZAYERI, S.M.H.M; AMANI, R. Nutritional knowledge, attitudes and practices of bodybuilding trainers in ahwaz, Iran. Pakistan Journal of Nutrition, v. 3, n. 4, p. 228-23I, 2004.

JUZWIAK, C. R; ANCONA-LOPEZ, F. Evaluation of nutrition knowledge and dietary recommendations by coaches of adolescent brazilian athletes. International Journal of Sport Nutrition and Exercise Metabolism, Champaign, v. I 4, n. 2, p. 222-235, apr.2004.

MARINARO, L. M. Nutrition competency of certified athletic trainers. 2008. 92 f. Dissertação (Mestrado em Ciência, Nutrição e Dietética) - Universidade de Akron, Ohio, 2008.

MORRISON, L. J.; GIZIS F; SHORTER, B. Prevalent use of dietary supplements among people who exercise at a commercial gym. International Journal of Sport Nutrition and Exercise Metabolism, Champaign, v. I4, n. 4, p. 48I-492, ago. 2004.

OZDOGAN, Y.; OZCELIK, A. O. Evaluation of the nutrition knowledge of sports de- partment students of universities. Journal of the International Society of Sports Nutrition, Champaign, v. 8, n. I I, p. I-7, sept. 201 I. 
PEREIRA, J. M. O.; CABRAL, P. Avaliação dos conhecimentos básicos sobre nutrição de praticantes de musculação em uma academia da cidade de recife. Revista Brasileira de Nutrição Esportiva, São Paulo, v. I, n. I, p. 40-47, jan./fev. 2007.

PEREIRA, R. F.; LAJOLO, F. M.; HIRSCHBRUCH, M. D. Consumo de suplementos por alunos de academia de ginástica em São Paulo. Revista de Nutrição, Campinas, v. I6, n. 3, p. 265-272, jul./set. 2003.

PERIM, G. L.; ZUCAS, S. M. Conhecimentos nutricionais dos professores de Educação Física e Esportes: um estudo realizado no município de Londrina-Pr. Revista Brasileira de Atividade Física e Saúde, Londrina, v. 6, n. I, p. 67, 2001.

ROCKWELL, M. S.; NICHOLS-RICHARDSON, S. M.; THYE, F. W. Nutrition knowledge, opinions, and practices of coaches and athletic trainers at a division i university. International Journal of Sport Nutrition and Exercise and Metabolism, Champaign, v. I I , n. 2, p. I 74- I 85, june 200 I .

RODRIGUES, E. M; SOARES, F. P. T. P; BOOG, M. C. F. Resgate do conceito de aconselhamento no contexto do atendimento nutricional. Revista de Nutrição, Campinas, v. I 8, n. I, p. I19-128, jan./fev. 2005.

ROPELATO, F. F; RAVAZZANI, E. D. A. Percepção de acadêmicos de Educação Física em relação aos suplementos alimentares. Cadernos da Escola de Saúde, Curitiba, v. I, n. 5, p. $81-92,2011$.

ROSENBLOOM, C. A.; JONNALAGADDA, S. S.; SKINNER, R. Nutrition knowledge of collegiate athletes in a division i national collegiate Athletic Association Institution. Journal of the American Dietetic Association, Chicago, v. 102, n. 3, p. 4l8-420, 2002.

ROWLAND, D.; ARKKELIN, D; CRISLER, L. Computer-based data analysis: using SPSSx in the social and behavioral sciences. Chicago: Nelson-Hall, 1991.

SANTOS, M. A. A.; SANTOS, R. P. Uso de suplementos alimentares como forma de melhorar a performance nos programas de atividade física em academias de ginástica. Revista paulista de Educação Física, São Paulo, v. I6, n. 2, p. |74- |85, jul./dez. 2002.

SCOFIELD, D. E; UNRUH, S. Dietary supplement use among adolescent athletes in central Nebraska and their sources of information. Journal of Strength and Conditioning Research, Colorado Springs, v. 20, n. 2, p. 452- 455, may 2006.

STREINER, D. Starting at the beginning: an introduction to coefficient alpha and internal consistency. Journal of Personality Assessment, Hillsdale, v. 80, n. I , p. 99- I03, feb. 2003.

TOLENTINO, F. M. et al. A nutrição esportiva no curso de educação física: verificação sobre o conhecimento dos acadêmicos. Educação Física em Revista, Avaré, v. 4, n. 3, p. I-8, set./ dez. 2010.

TORRES-MCGEHEE, T. M. et al. Sports nutrition knowledge among collegiate athletes, coaches, athletic trainers, and strength and conditioning specialists. Journal of Athletic Training, Dallas, v. 47, n. 2, p. 205-211, apr. 2012. 
UNIVERSIDADE OF MASSEY. Institute of Food, Nutrition and Human Health. Assesment of nutritional knowledge and food skills in talented adolescent athletes. Palmerston Norte, 2010.

ZAWILA, L. G.; STEIB, C. M.; HOOGENBOOM, B. The female collegiate cross-country runner: nutritional knowledge and attitudes. Journal of Athletic Training, Dallas, v. 38, n. I, p. 67-74, mar. 2003.

ZINN, C. Nutrition knowledge of New Zealand Premier Club rugby coaches. 159p. Dissertação (Mestrado em Ciências da Saúde) - Universidade de Auckland, Auckland, 2004.

Recebido em: 4 jun. 2012

Aprovado em: 20 abr. 2013

Endereço para Correspondência:

Raquel Simões Mendes Netto

Núcleo de Nutrição, Centro de Ciências Biológicas e da Saúde, Universidade Federal de Sergipe. Av. Marechal Rondon, s/n Jardim Rosa Elze, CEP 49100-000 Cidade Universitária Professor "José Aloísio de Campos", São Cristóvão-SE. 


\section{ANEXO I}

\section{Questionário sobre Conhecimentos em Nutrição TEMA: CONHECIMENTOS GERAIS}

I) Você acha que estes alimentos apresentam alto ou baixo conteúdo de CARBOIDRATOS? (marque uma alternativa por alimento)

$\begin{array}{cccc} & \text { Alto } & \text { Baixo } & \text { Não sei / Não tenho } \\ \text { certeza }\end{array}$

2) Você acha que estes alimentos apresentam alto ou baixo conteúdo de PROTEÍNA? (marque uma alternativa por alimento)

$\begin{array}{cccc} & \text { Alto } & \text { Baixo } & \text { Não sei / Não tenho } \\ \text { Frango } & (\text { ) } & (\text { ) } & (\text { ) } \\ \text { Feijão Cozido } & (\text { ) } & (\text { ) } & (\text { ) } \\ \text { Frutas } & (\text { ) } & (\text { ) } & (\text { ) } \\ \text { Margarina } & (\text { ) } & (\text { ) } & (\text { ) } \\ \text { Cereal matinal } & (\text { ) } & (\text { ) } & (\text { ) } \\ \text { Amendoim } & (\text { ) } & (\text { ) } & (\text { ) }\end{array}$

3) Você acha que estes alimentos apresentam alto ou baixo conteúdo de GORDURA? (marque uma alternativa por alimento)

$\begin{array}{cccc} & \text { Alto } & \text { Baixo } & \text { Não sei / Não tenho certeza } \\ \text { Abacate } & (\text { ) } & (\text { ) } & (\text { ) } \\ \text { Feijão Cozido } & (\text { ) } & (\text { ) } & (\text { ) } \\ \text { Macarrão } & (\text { ) } & (\text { ) } & (\text { ) } \\ \text { Ricota } & (\text { ) } & (\text { ) } & (\text { ) } \\ \text { Amendoim } & (\text { ) } & (\text { ) } & (\text { ) }\end{array}$




$\begin{array}{clll}\text { Pão branco } & (\text { ) } & (\text { ) } & (\text { ) } \\ \text { Mel } & (\text { ) } & (\text { ) } & (\text { ) } \\ \text { Queijo cheddar } & (\text { ) } & (\text { ) } & (\text { ) }\end{array}$

4) Você acha que estes alimentos apresentam alto ou baixo conteúdo de GORDURA SATURADA ?(marque uma alternativa por alimento).

$\begin{array}{cccc} & \text { Alto } & \text { Baixo } & \text { Não sei / Não tenho certeza } \\ \text { Manteiga } & (\text { ) } & (\text { }) & (\text { ) } \\ \text { Óleo de canola } & (\text { ) } & (\text { ) } & (\text { ) } \\ \text { Leite integral } & (\text { ) } & (\text { ) } & (\text { ) } \\ \text { Carne vermelha } & (\text { ) } & (\text { ) } & (\text { ) } \\ \text { Salmão } & (\text { ) } & (\text { ) } & (\text { ) } \\ \text { Chocolate } & (\text { ) } & (\text { ) } & (\text { ) }\end{array}$

5) Você acha que estes alimentos apresentam alto ou baixo conteúdo de COLESTEROL ?( marque uma alternativa por alimento).

$\begin{array}{cccc} & \text { Alto } & \text { Baixo } & \text { Não sei / Não tenho certeza } \\ \text { Carne vermelha } & (\text { ) } & (\text { ) } & (\text { ) } \\ \text { Ovos } & (\text { ) } & (\text { ) } & (\text { ) } \\ \text { Peixe } & (\text { ) } & (\text { ) } & (\text { ) } \\ \text { Margarina } & (\text { ) } & (\text { ) } & (\text { ) } \\ \text { Leite integral } & (\text { ) } & (\text { ) } & (\text { ) }\end{array}$

Marque as seguintes alternativas em verdadeiro, falso ou não sei/não tenho certeza

\begin{tabular}{|l|l|l|l|}
\hline 6) A principal fonte de energia do músculo é a proteína & (V) & (F) & (NS) \\
\hline $\begin{array}{l}\text { 7) As fibras ajudam a diminuir a constipação, os níveis de colesterol } \\
\text { sanguíneos e a prevenir o câncer }\end{array}$ & $(V)$ & (F) & (NS) \\
\hline $\begin{array}{l}\text { 8) No máximo I 5\% das calorias da dieta devem ser provenientes } \\
\text { de gordura }\end{array}$ & $(V)$ & (F) & (NS) \\
\hline 9) Vitaminas e minerais são boas fontes de energia & $(V)$ & (F) & (NS) \\
\hline 10) O consumo de álcool afeta a utilização e absorção dos nutrientes & V) & (F) & (NS) \\
\hline 1 1) Uma dieta rica em carboidrato ajuda a poupar a proteína do corpo & (V) & (F) & (NS) \\
\hline
\end{tabular}




\section{TEMA: NUTRIÇÃO ESPORTIVA}

Marque as seguintes alternativas em verdadeiro, falso ou não sei/não tenho certeza:

\begin{tabular}{|c|c|c|c|}
\hline $\begin{array}{l}\text { 2) Uma pessoa fisicamente ativa e que possua uma dieta nutricionalmente adequada } \\
\text { de melhorar sua performance consumindo uma maior quantidade de nutrientes. }\end{array}$ & $(\mathrm{V})$ & $(F)$ & (NS) \\
\hline I3) Pular refeições é justificável quando é preciso perder peso rapidamente. & $(\mathrm{V})$ & $(F)$ & (NS) \\
\hline $\begin{array}{l}\text { nutricionalmente adequada para atletas é ingerir uma variedade de } \\
\text { os. }\end{array}$ & $(V)$ & $(F)$ & (NS) \\
\hline 15) Durante o exerć́cio, a sede é um indicador adequado de necessidade de líquidos. & $(\mathrm{V})$ & $(\mathrm{F})$ & (NS) \\
\hline $\begin{array}{l}\text { 16) Algumas comidas( por ex:abacaxi), possuem grar } \\
\text { perda de peso já que podem ajudar na queima da go }\end{array}$ & $(\mathrm{V})$ & $(\mathrm{F})$ & (NS) \\
\hline 17) O tipo de comida que um atleta ingere influencia no seu desempenho & $(V)$ & $(F)$ & (NS) \\
\hline $\begin{array}{l}\text { carboidratos devem ser provenientes } \\
\text { ães. }\end{array}$ & $(\mathrm{V})$ & $(F)$ & (NS) \\
\hline $\begin{array}{l}\text { 19) } \bigcirc \text { que um atleta ingere somente é importante se ele pretende ganhar ou perder } \\
\text { peso. }\end{array}$ & $(\mathrm{V})$ & $(F)$ & (NS) \\
\hline $\begin{array}{l}\text { 20) Para que o ganho de massa muscular ocorra, a proteína é o nutriente mais importante } \\
\text { para se aumentar na dieta. }\end{array}$ & $(\mathrm{V})$ & $(F)$ & (NS) \\
\hline $\begin{array}{l}\text { ) Durante o treinamento de força, o consumo de repositores eletrolíticos é melhor } \\
\text { que o consumo de água }\end{array}$ & $(V)$ & $(F)$ & (NS) \\
\hline
\end{tabular}

Se um praticante de musculação com peso adequado tem como objetivo ganhar massa muscular, ele deve:

\begin{tabular}{|l|l|l|l|}
\hline 22) Ingerir shake de proteína(whey) durante o treino. & $(V)$ & (F) & (NS) \\
\hline $\begin{array}{l}\text { 23) Alimentar-se imediatamente após o treino ( Por exemplo: vitamina de banana com } \\
\text { aveia + sanduiche de frango). }\end{array}$ & $(V)$ & (F) & (NS) \\
\hline 24) Optar por suplementos termogênicos a base de cafeína. & $(V)$ & (F) & (NS) \\
\hline
\end{tabular}

Marque as seguintes alternativas em verdadeiro, falso ou não sei/não tenho certeza:

\begin{tabular}{|l|l|l|l|}
\hline 25) A creatina traz mais benefícios a um atleta que queira aumentar a sua força. & $(V)$ & (F) & (NS) \\
\hline $\begin{array}{l}\text { 26) A suplementação com creatina faz mais efeito quando seus estoques naturais } \\
\text { estão baixos. }\end{array}$ & V) & (F) & (NS) \\
\hline 27) O principal mecanismo da creatina é que ela aumenta o metabolismo dos lipídios. & $(V)$ & (F) & (NS) \\
\hline $\begin{array}{l}\text { 28) A creatina é mais eficaz para atletas que queiram melhorar seu desempenho físico } \\
\text { em exercícios de resistência. }\end{array}$ & (V) & (F) & (NS) \\
\hline $\begin{array}{l}\text { 29) Os suplementos multivitamínicos devem ser tomados pela maioria dos atletas, } \\
\text { mesmo consumindo uma dieta adequada e balanceada. }\end{array}$ & (V) & (F) & (NS) \\
\hline
\end{tabular}




\begin{tabular}{|c|c|c|c|}
\hline $\begin{array}{l}\text { 30) Atletas precisam de uma maior quantidade de vitaminas do que indivíduos se- } \\
\text { dentários. }\end{array}$ & $(V)$ & $(\mathrm{F})$ & (NS) \\
\hline 3I) A cafeína melhora o desempenho em exercícios de resistência. & $(V)$ & $(\mathrm{F})$ & (NS) \\
\hline $\begin{array}{l}\text { 32) O consumo de maltodextrina durante o treino deve ser evitado, pois esta pode } \\
\text { favorecer o ganho de gordura corporal. }\end{array}$ & $(\mathrm{V})$ & $(\mathrm{F})$ & $(\wedge$ \\
\hline ) Suplementos protéicos em excesso podem causar sobrecarg & $(V)$ & $(\mathrm{F})$ & $(\mathrm{N}$ \\
\hline
\end{tabular}

Entregue seu questionário.

Suas informações serão mantidas confidenciais. Obrigado por participar! 